\title{
Elevated expression of yes-associated protein is associated with the malignant status and prognosis of laryngeal squamous cell carcinoma
}

\author{
CHUNCHEN PAN ${ }^{1,2}$, ZHIHUI DU $^{1}$, ZHAOGEN CAI $^{3}$, YUN LIU $^{1}$, YANBO SUN $^{1}$, JIN CHEN $^{1}$, TING TONG $^{1}$, \\ QINGGUO CHEN ${ }^{1}$, LIANGQIANG ZHOU ${ }^{1}$, DAN BING ${ }^{1}$, YANLING TAO ${ }^{1}$ and HANQI CHU ${ }^{1}$ \\ ${ }^{1}$ Department of Otolaryngology-Head and Neck Surgery, Tongji Hospital, Tongji Medical College, Huazhong \\ University of Science and Technology, Wuhan, Hubei 430030; ${ }^{2}$ Department of Otolaryngology-Head and \\ Neck Surgery, Anhui Provincial Hospital, Anhui Medical University, Hefei, Anhui 230000; ${ }^{3}$ Department of \\ Pathology, The First Hospital Affiliated to Bengbu Medical College, Bengbu, Anhui 233000, P.R. China
}

Received February 9, 2016; Accepted February 20, 2017

DOI: $10.3892 / \mathrm{mmr} .2017 .7187$

\begin{abstract}
Previous studies have demonstrated that elevated yes-associated protein (YAP) expression is associated with tumor aggression and poor prognosis in various types of human cancer. However, the clinicopathological significance and the prognostic value of YAP in laryngeal squamous cell carcinoma (LSCC) is unknown. The aim of the present study was to identify the expression pattern and prognostic significance of YAP in patients with LSCC. YAP mRNA and protein expression levels were examined in fresh and archived LSCC samples using the reverse transcription-quantitative polymerase chain reaction, immunohistochemistry (IHC) and western blotting. The association between YAP expression levels with the malignant status and prognosis of patients with LSCC was analyzed. Upregulated protein and mRNA expression levels of YAP were detected in LSCC tissues compared with paired healthy surgical margin tissues. Positive expression of YAP was identified in 84/121 (69.4\%) LSCC tissues and in 4/30 (13.3\%) healthy surgical margin tissues by IHC. Positive YAP protein expression was significantly associated with clinical stage, TNM classification, lymph node metastasis and differentiated degree. Patients with positive YAP expression exhibited a significantly decreased overall survival time compared with patients with negative YAP expression $(\mathrm{P}=0.0002)$. Multivariate analysis indicated that the level of YAP expression was an independent prognostic factor for poor survival in patients with LSCC $(\mathrm{P}=0.012)$. In conclusion, the
\end{abstract}

Correspondence to: Dr Hanqi Chu, Department of Otolaryngology-Head and Neck Surgery, Tongji Hospital, Tongji Medical College, Huazhong University of Science and Technology, 1095 Jiefang Avenue, Wuhan, Hubei 430030, P.R. China E-mail: qi7chu@163.com

Key words: yes-associated protein, immunohistochemistry, laryngeal squamous cell carcinoma, prognosis, biomarker expression level of YAP was significantly increased in LSCC and associated with the malignant status of LSCC. Therefore, YAP may represent a novel biomarker for predicting the prognosis of patients with LSCC.

\section{Introduction}

Laryngeal squamous cell carcinoma (LSCC), accounting for 95\% of laryngeal cancers, has the second highest incidence of all head and neck squamous cell carcinomas (1). The morbidity of LSCC is increasing annually (1). LSCC occurrence and development is a multi-factor, multi-stage process, and environmental and genetic factors serve vital roles in its etiology. However, the underlying molecular mechanisms of LSCC remain largely unknown. Although the diagnosis and treatment of LSCC have improved, the overall survival (OS) time of patients with LSCC has not significantly improved over previous decades $(2,3)$. For physicians, predicting patient with LSCC outcome and making therapeutic decisions based only on the Tumor Node Metastasis (TNM) Classification of Malignant Tumors is a limited (4). Therefore, it is urgent to develop clinically useful markers to facilitate the diagnosis and prognosis of LSCC, which will contribute to increased survival rates.

Yes-associated protein (YAP) is the mammalian ortholog of Drosophila Yorkie and the nuclear effector downstream target of the Hippo signaling pathway (5). YAP activity is regulated by phosphorylating the conserved serine 127 residue and subcellular localization via the Hippo signaling pathway (6). As a transcriptional co-activator, YAP interacts with TEA domain family member and other transcription factors to regulate the expression of target genes involved in the regulation of cell growth, proliferation, and apoptosis (7).

YAP has been demonstrated to be a candidate oncogene in the human chromosome 11q22 amplicon (8), and hyperactivation of YAP is widespread in cancers (9). YAP is overexpressed in human lung, ovarian, gastric, pancreatic, bladder, colorectal, prostate carcinomas and brain malignancies (9-12), and has been identified to be an independent prognostic marker 
for hepatocellular (13), gastric (14), bladder (15), lung (16), bile duct (17), ovarian (18), and esophageal (19) carcinomas. It was reported that YAP is strongly expressed at the tumor invasive front and in metastatic head and neck squamous cell carcinoma, and is associated with lymph node metastasis (LNM) (20). However, the YAP expression pattern and its clinical and prognostic significance in LSCC remains to be completely elucidated. The purpose of the present study was to evaluate the expression and potential prognostic significance of YAP in patients with LSCC.

\section{Materials and methods}

Patients. Fresh LSCC tumor and paired adjacent healthy surgical margin tissue samples ( $n=30 /$ group) were collected from the First Affiliated Hospital of Bengbu Medical College (Bengbu, China), between July 2013 and September 2015. All samples were immediately preserved in liquid nitrogen. Archived paraffin-embedded tissues were collected, including 91 cases of LSCC from the Pathology Department of the Affiliated Union Hospital of Fujian Medical University (Fujian, China) between July 2008 and September 2010. None of the cases had undergone chemotherapy or radiotherapy. The medical records of these patients were reviewed to assess patient characteristics, including age, sex, smoking, alcohol, tumor location, clinical stage, TNM classification, LNM, differentiated degree and final status on the last follow-up examination. The cases aged from 39 to 80 years (mean age 60.2 years) and the clinical and pathological information of all samples is presented in Table I.

In the patients with LSCC, smoking habits were defined as non-smokers (<100 cigarettes in their lifetime) or smokers. Alcohol consumption was defined as non-drinker or drinker (more than $200 \mathrm{ml} /$ day). The mean observation time for overall survival was 60 months for patients still alive at the time of analysis, and ranged from 1-60 months. A total of 38 patients $(41.76 \%)$ succumbed to disease during follow-up. Informed consent was obtained from each patient prior to surgery and the study was approved by the Institute Research Ethics Committee of Tongji Medical College, Huazhong University of Science and Technology, Hubei, China.

Immunohistochemistry (IHC) and scoring. IHC was performed according to previously described protocols (21). Each paraffin-embedded tissue sample was sectioned into 4- $\mu \mathrm{m}$ thick continuous slices. Paraffin-embedded sections were deparaffinized in xylene, rehydrated in a descending ethanol series and double distilled water. High pressure antigenic retrieval was performed in citrate buffer $(\mathrm{pH} \mathrm{6.0)}$ and the samples were boiled for $2 \mathrm{~min}$. Following antigen retrieval, sections were treated with $3 \%$ hydrogen peroxide and $1 \%$ bovine serum albumin (Wuhan Boster Biological Technology, Ltd., Wuhan, China) to block endogenous peroxidase activity and non-specific binding. The sections were incubated with a mouse monoclonal anti-YAP antibody (catalog no. AT4556a; Abgent, Inc., San Diego, CA, USA; dilution 1:500) overnight at $4^{\circ} \mathrm{C}$. Subsequently, sections were washed three times with $0.1 \mathrm{mM}$ PBS and incubated with a horseradish peroxidase conjugated secondary antibody (cat. no. BA1025; 1:200; Boster Biotechnology, Inc., Wuhan, China)
Table I. Correlations between YAP expression with clinical pathological characteristics in 121 LSCC cases.

\begin{tabular}{|c|c|c|c|}
\hline \multirow[b]{2}{*}{ Factors } & \multirow[b]{2}{*}{ Cases } & \multicolumn{2}{|c|}{ YAP } \\
\hline & & Positive; N (\%) & P-value \\
\hline \multicolumn{4}{|l|}{ Age (years) } \\
\hline$\geq 60$ & 68 & $49(72.0)$ & 0.476 \\
\hline$<60$ & 53 & $35(66.0)$ & \\
\hline \multicolumn{4}{|l|}{ Sex } \\
\hline Male & 117 & $81(69.2)$ & 0.575 \\
\hline Female & 4 & $3(75)$ & \\
\hline \multicolumn{4}{|l|}{ Location } \\
\hline Non-glottic & 48 & $34(70.8)$ & \\
\hline Glottic & 73 & $50(68.5)$ & 0.785 \\
\hline \multicolumn{4}{|l|}{ Smoking } \\
\hline Yes & 84 & $60(71.4)$ & 0.470 \\
\hline No & 37 & $24(63.9)$ & \\
\hline \multicolumn{4}{|l|}{ Alcohol } \\
\hline Yes & 42 & $29(71.9)$ & 0.948 \\
\hline No & 79 & 55 (61.7) & \\
\hline \multicolumn{4}{|l|}{ Clinical stage } \\
\hline I-II & 67 & $39(58.2)$ & 0.003 \\
\hline III-IV & 54 & $45(83.3)^{\mathrm{a}}$ & \\
\hline \multicolumn{4}{|l|}{ TNM } \\
\hline $\mathrm{T} 1-\mathrm{T} 2$ & 66 & $36(54.5)$ & $<0.001$ \\
\hline T3-T4 & 55 & $48(87.3)^{\mathrm{a}}$ & \\
\hline \multicolumn{4}{|l|}{ LNM } \\
\hline Absent & 86 & $52(60.5)$ & 0.001 \\
\hline Present & 35 & $32(91.4)^{\mathrm{a}}$ & \\
\hline \multicolumn{4}{|l|}{ Differentiation } \\
\hline High & 76 & $46(59.0)$ & 0.001 \\
\hline Moderate/low & 45 & $38(88.4)^{\mathrm{a}}$ & \\
\hline
\end{tabular}

${ }^{\text {aP }}<0.05$. LNM, lymph node metastasis; TNM, TNM Classification of Malignant Tumors; YAP, yes-associated protein; LSCC, laryngeal squamous cell carcinoma.

for $60 \mathrm{~min}$ at room temperature. Sections were stained with 3,3-diaminobenzidine for 2-5 min, rinsed with 0.1 mM PBS, and counterstained with hematoxylin. Sections were viewed under a light microscope.

The final score of each sample was scored independently by two pathologists and assessed by adding the scores for the intensity and extent of staining. YAP expression was graded according to the distribution, intensity, and percentage of positive cells as described previously $(9,15)$. Absence of reactivity was graded as negative. With regard to cytoplasmic distribution, weak cytoplasmic reactivity was considered as low expression regardless of extent. Strong cytoplasmic reactivity with $<50 \%$ positive cells was graded as low expression. Otherwise it was graded as high expression. With regard to nuclear distribution, nuclear expression in $<10 \%$ cells was graded as low and $>10 \%$ cells was graded as high. Samples 
with low or high YAP staining were classified as YAP positive expression.

$R N A$ extraction and reverse transcription-quantitative polymerase chain reaction $(R T-q P C R)$. Total RNA from fresh samples was extracted using TRIzol ${ }^{\circledR}$ reagent (Invitrogen; Thermo Fisher Scientific, Inc., Waltham, MA, USA) according to the manufacturer's protocol, and cDNA was synthesized using ReverTra Ace ${ }^{\circledR}$ (Toyobo Co., Ltd., Osaka, Japan). qPCR was performed using SYBR ${ }^{\circledR}$ Green reagent (Toyobo Co., Ltd.) on a LightCycler ${ }^{\circledR} 480$ II system (Roche Diagnostics, Basel, Switzerland). Primers specific for YAP were purchased from Sangon Biotech Co., Ltd. (Shanghai, China). The sequences of primers used were as follows: Forward, 5'-ACCCAGAAG ACTGTGGATGG-3' and reverse, 5'-TTCTAGACGGCAGGT CAGGT-3' for GAPDH; forward, 5'-CTTCCTTGTCCATTG CTGCT-3' and reverse, 5'-ATCCAAACAGGCTCACTTCC-3' for YAP. The following amplification steps were used: Initial denaturation for $30 \mathrm{sec}$ at $95^{\circ} \mathrm{C}, 40$ cycles of denaturation for $5 \mathrm{sec}$ at $95^{\circ} \mathrm{C}$, annealing for $10 \mathrm{sec}$ at $60^{\circ} \mathrm{C}$, and extension for $15 \mathrm{sec}$ at $72^{\circ} \mathrm{C}$. Each sample was run in triplicate, and mean values were calculated. The relative gene expression was calculated using the $2^{-\Delta \Delta C q}$ method (22).

Western blotting. Tissue (100 mg) was ground into powder in liquid nitrogen. Frozen tissues were lysed in lysis buffer (cat. no. P0013C; Beyotime Institute of Biotechnology, Jiangsu, China), and protein concentrations were measured using a Bicinchoninic Acid Protein Assay kit (cat. no. P0009; Beyotime Institute of Biotechnology) and preserved at $-20^{\circ} \mathrm{C}$. Proteins $(20 \mu \mathrm{g})$ were separated by $10 \%$ SDS-PAGE at $20 \mathrm{~V}$ for $60 \mathrm{~min}$ and subsequently transferred onto nitrocellulose filter membranes. The membranes were incubated with the anti-YAP primary antibody (cat. no. AT4556a; 1:1,000; Abgent, Inc., San Diego, CA, USA) overnight at $4^{\circ} \mathrm{C}$ and were subsequently incubated with Goat anti-Mouse IgG $(\mathrm{H}+\mathrm{L})$ Cross-Adsorbed Secondary Antibody, Horseradish Peroxidase conjugate (cat. no. A16072; 1:3,000; Thermo Fisher Scientific, Inc.) for $1 \mathrm{~h}$ at $37^{\circ} \mathrm{C}$. Bands were visualized using an Enhanced Chemiluminescence detection system (ChemiDoc MP; Bio-Rad Laboratories, Inc., Hercules, CA, USA) and Image Lab software (version 4.0; Bio-Rad Laboratories, Inc.). All tests were repeated at least three times (21).

Statistical analysis. Data are presented as the mean \pm standard deviation and were statistically analysed using SPSS software version 19.0 (SPSS, Inc., Chicago, IL, USA). The Student's t-test was applied to assess differential mRNA expressions of YAP in LSCC tissues compared with paired healthy surgical margin tissues. The $\chi^{2}$ test was used to analyze the correlation between YAP expression and the clinicopathological parameters of LSCC patients. For analysis of overall survival, Kaplan-Meier survival curves were constructed for groups based on univariate predictors, and differences between groups were tested with the log-rank test. Univariate and multivariate survival analyses were performed using the likelihood ratio test of the stratified Cox proportional hazards model. $\mathrm{P}<0.05$ was considered to indicate a statistically significant difference.
Table II. Immunohistochemistry of YAP protein expression levels.

\begin{tabular}{lcrcr}
\hline & & \multicolumn{2}{c}{ YAP } \\
\cline { 3 - 4 } Tissue \\
\cline { 3 - 4 } $\begin{array}{l}\text { type } \\
\text { LSCC }\end{array}$ & Cases & $\begin{array}{c}\text { Positive } \\
(\%)\end{array}$ & $\begin{array}{c}\text { Negative } \\
(\%)\end{array}$ & P-value \\
\hline Healthy & 121 & $84(69.4)$ & $37(30.6)$ & $<0.001$ \\
\hline
\end{tabular}

YAP, yes-associated protein; LSCC, laryngeal squamous cell carcinoma.

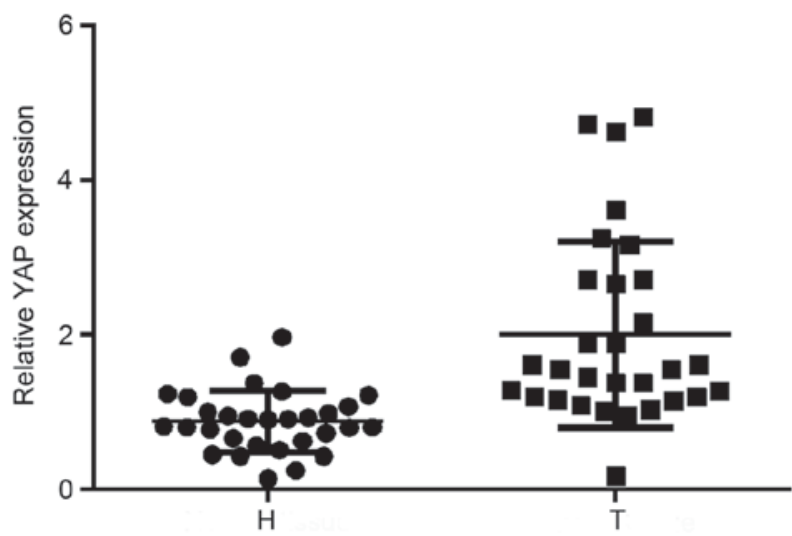

Figure 1. YAP mRNA expression levels. YAP mRNA levels were significantly increased in LSCC tissues compared with adjacent healthy surgical margin tissues, as assessed by reverse transcription-quantitative polymerase chain reaction. Data are presented as the mean \pm standard deviation ( $n=30$ /group). $\mathrm{P}<0.001$. YAP, yes-associated protein; LSCC, laryngeal squamous cell carcinoma; $\mathrm{T}$, tumor tissue; H, paired adjacent healthy surgical margin tissue.

\section{Results}

YAP overexpression in LSCC tissues. RT-qPCR was performed in 30 pairs of fresh cancer and paired healthy surgical margin tissues from patients with LSCC to measure the expression of YAP mRNA. YAP mRNA expression levels were significantly elevated with a mean increase in LSCC tissues by 2.83 -fold in comparison to paired healthy surgical margin tissues $(\mathrm{P}<0.001$; Fig. 1). Western blotting was performed to measure protein expression levels of YAP, and elevated expression of YAP was detected in 27/30 tumor tissues compared with healthy tissues (Fig. 2).

IHC analysis revealed that YAP was present in 84/121 $(69.4 \%)$ of LSCC tissues and in 4/30 (13.3\%) of healthy tissues (Table II). Furthermore, YAP subcellular localization was primarily accumulated in the nucleus with a reduced cytoplasmic presence in the majority of LSCC cases (Fig. 3A-H). By contrast, YAP staining was rarely observed in the nuclei of healthy tissues (Fig. 3I-J).

Association between clinicopathological characteristics and expression of YAP in patients with LSCC. The association between clinicopathological characteristics and YAP 
Table III. Univariate and multivariate Cox regression analyses of overall survival duration.

\begin{tabular}{|c|c|c|c|c|}
\hline \multirow[b]{2}{*}{ Parameter } & \multicolumn{2}{|r|}{ Univariate analysis } & \multicolumn{2}{|c|}{ Multivariate analysis } \\
\hline & P-value & $95 \% \mathrm{CI}$ & P-value & $95 \% \mathrm{CI}$ \\
\hline $\begin{array}{l}\text { Sex } \\
\text { female vs. male }\end{array}$ & 0.137 & $0.35710 .01727-1.725$ & & \\
\hline $\begin{array}{l}\text { Age } \\
<60 \text { vs. } \geq 60 \text { years }\end{array}$ & 0.7107 & $1.129 \quad 0.5932-2.154$ & & \\
\hline $\begin{array}{l}\text { Smoking } \\
\text { No vs. yes }\end{array}$ & 0.7517 & $0.89830 .4496-1.776$ & & \\
\hline $\begin{array}{l}\text { Alcohol } \\
\text { No vs. yes }\end{array}$ & 0.1843 & $0.62490 .2638-1.287$ & & \\
\hline $\begin{array}{l}\text { Localization } \\
\text { Glottic vs. non-glottic }\end{array}$ & 0.9347 & $0.89350 .4448-1.795$ & & \\
\hline $\begin{array}{l}\text { Differentiated degree } \\
\text { High vs. low or moderate }\end{array}$ & $<0.0001$ & $\begin{array}{lll}0.371 & 0.2093-0.4899\end{array}$ & $<0.0001$ & $1.9830 .060-0.316$ \\
\hline $\begin{array}{l}\text { Clinical stage } \\
\text { I-II vs. III-IV }\end{array}$ & $<0.0001$ & $0.32020 .1994-0.5142$ & 0.002 & $1.5880 .266-0.694$ \\
\hline $\begin{array}{l}\text { TNM classification } \\
\text { T1-T2 vs. T3-T4 }\end{array}$ & 0.0035 & $0.43720 .2019-0.7976$ & 0.031 & $0.7451 .330-3.336$ \\
\hline $\begin{array}{l}\text { LNM } \\
\text { Absent vs. present }\end{array}$ & $<0.0001$ & $0.23810 .0558-0.2943$ & 0.028 & $1.0280 .559-3.159$ \\
\hline $\begin{array}{l}\text { YAP } \\
\text { Negative vs. positive }\end{array}$ & 0.0002 & $0.22120 .1503-0.5470$ & 0.012 & $0.8500 .216-0.831$ \\
\hline
\end{tabular}

HR, hazard ratio; CI, confidence interval; LNM, lymph node metastasis; TNM, TNM Classification of Malignant Tumors; YAP, yes-associated protein.

A

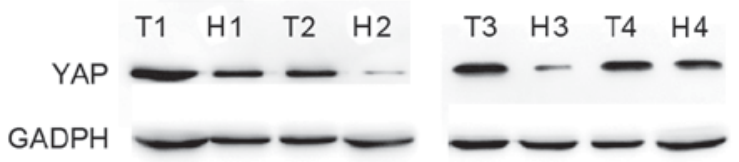

B

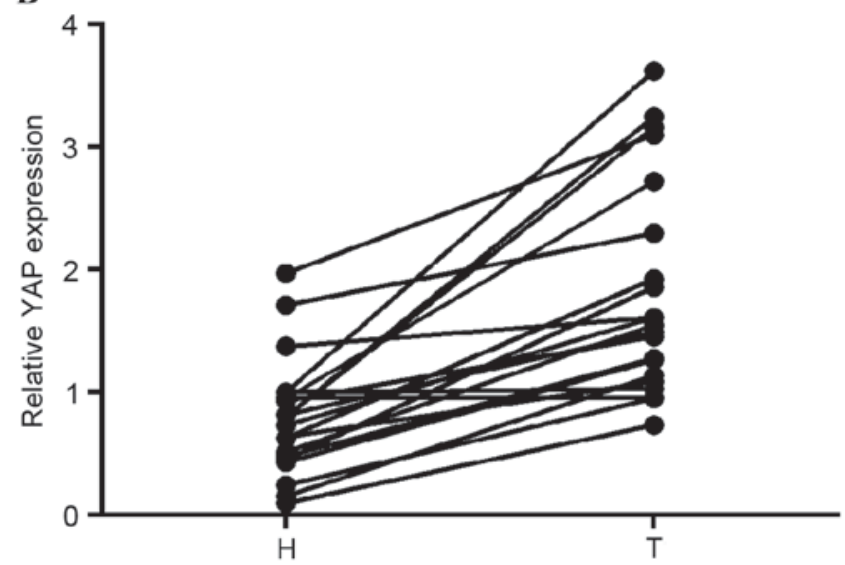

Figure 2.Protein expression levels of YAP in LSCC tissues. (A) Representative western blot images and (B) quantification of protein expression levels in LSCC tissues compared with adjacent healthy tissues. GADPH served as an internal control. T, tumor tissue; $\mathrm{H}$, paired adjacent healthy surgical margin tissue; YAP, yes-associated protein; LSCC, laryngeal squamous cell carcinoma. expression levels in 121 patients with LSCC are summarized in Table I. YAP was positively associated with clinical stage (I-II vs. III-IV, $\mathrm{P}<0.001)$, TNM stage (T1-T2 vs. T3-T4, $\mathrm{P}=0.003$ ), LNM (absent vs. present, $\mathrm{P}=0.001$ ), and differentiated degree (high vs. low or middle, $\mathrm{P}=0.001$ ) in LSCC. However, no significant differences were observed between LSCC patients with different ages, genders, smoking and alcohol habits, and tumor locations ( $\mathrm{P}>0.05$; Table I).

Prognosis and multivariate analysis. In 91 LSCC cases with prognosis information, patients with positive YAP protein expression exhibited decreased survival rates compared with those with negative YAP expression (5-year survival rate, $82.86 \%$ vs. $42.54 \%$, respectively; $\mathrm{P}<0.001$; Fig. 4). Clinical stage, TNM classification, LNM, differentiated degree and YAP protein expression were significantly associated with OS following univariate analysis $(\mathrm{P}<0.05$; Table III).

Selection of covariates for inclusion in the multivariate models was based on a univariate value of $\mathrm{P}<0.05$ for each respective association. Clinical stage, TNM classification, LNM, differentiated degree and YAP expression were determined to be independent prognostic factors for OS in LSCC patients by multivariate analyses using the Cox proportional hazards model. The level of positive expression of YAP was identified to be an independent prognostic factor for LSCC $(\mathrm{P}=0.012$; Table III). 

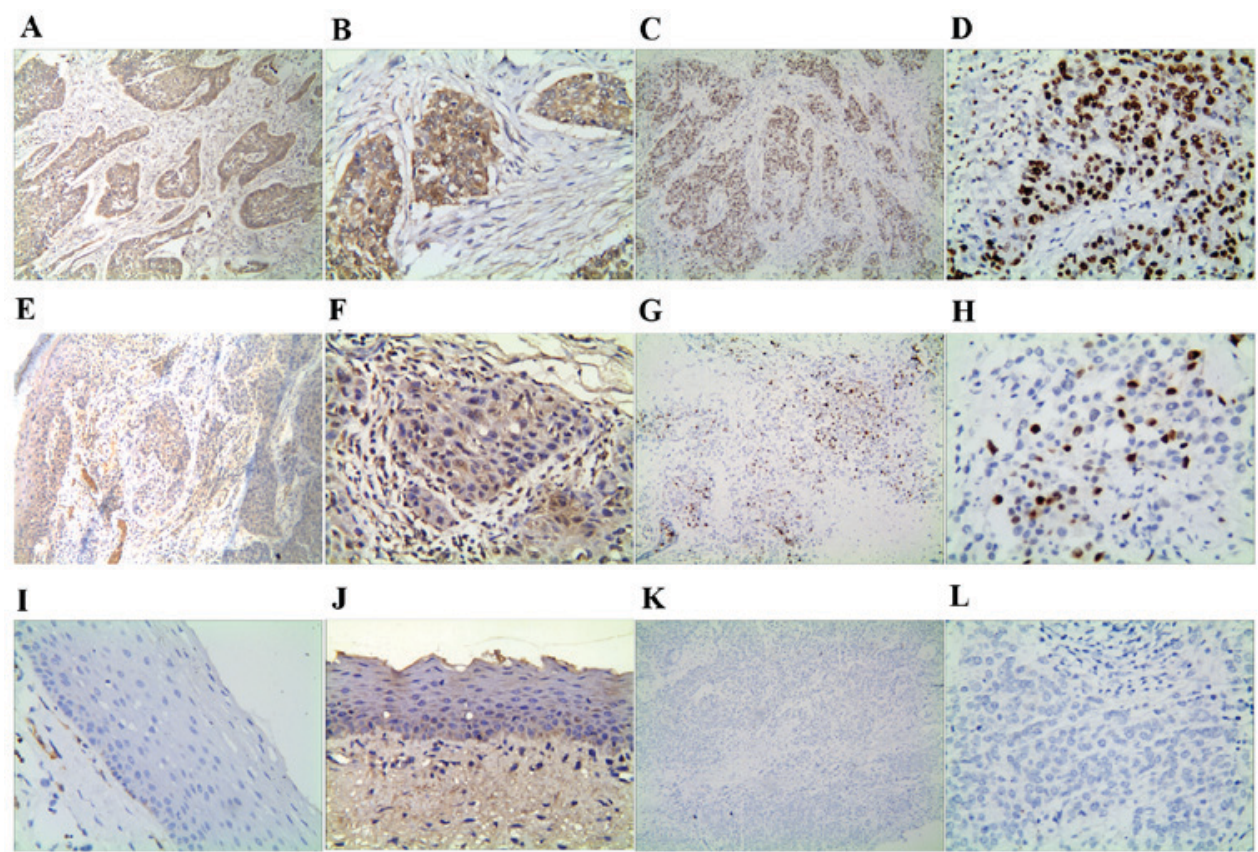

Figure 3. Immunohistochemical staining for YAP in primary human LSCC tissues. Representative images of LSCC tissues, demonstrating high YAP expression with both cytoplasm and nucleus present at magnification (A) x100 and (B) X400, high YAP expression primarily in the nucleus at magnification (C) x100 and (D) $\mathrm{x} 400$, low YAP expression with both cytoplasm and nucleus of tumor cells at magnification (E) x100 and (F) x400, and low expression of YAP protein mainly in the nucleus at magnification $(\mathrm{G}) \times 100$ and $(\mathrm{H}) \mathrm{x} 400$. Representative images of healthy surgical margin tissues, demonstrating (I) negative YAP expression and (J) low YAP expression. Negative control tissues were incubated with 1\% bovine serum albumin instead of a primary antibody (K) magnification, x100 and (L) magnification, x400. YAP, yes-associated protein; LSCC, laryngeal squamous cell carcinoma.

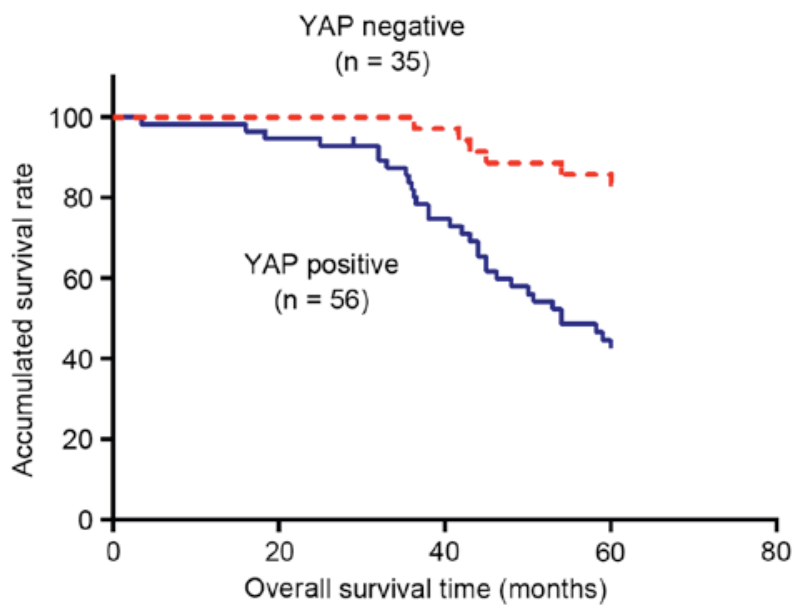

Figure 4. Positive YAP protein expression predicts an unfavorable prognosis The Kaplan-Meier method was used to determine the survival of 91 cases of laryngeal squamous cell carcinoma, and the log-rank test was used to compare five years' survival between the YAP negative and positive groups. $\mathrm{P}<0.001$. YAP, yes-associated protein.

\section{Discussion}

TNM staging and histopathological grading systems are well-established and useful prognostic indicators for LSCC (23). However, patients with identical clinical stage and/or pathological grade of laryngeal cancer frequently exhibit considerable variability in disease recurrence and survival (24). Currently, molecular biomarkers in conjunction with standard TNM and/or histopathological grading are considered to be a more effective strategy of prognosis.
YAP, as an oncogene, is a negatively regulated downstream target and major downstream effector of the Hippo signaling pathway (25). The Hippo signaling pathway is a conserved regulator of organ size, and controls multiple cellular functions that are central to tumorigenesis, including proliferation and apoptosis (12). The Hippo signaling pathway restricts tissue growth by inactivating the oncogenic activity of YAP/tafazzin (TAZ) in the nucleus and by enhancing its growth-suppressive activity in the cytoplasm (26). Dysregulation of the Hippo signaling pathway occurs in a broad range of human carcinomas, including lung (16), colorectal (27), breast (28), ovarian (29), esophageal squamous cell (19), gastric (14) and liver cancer (30). When Hippo signaling is absent or suppressed, unphosphorylated YAP and TAZ enter the nucleus, and induce transcription of genes involved in cell anti-apoptosis, survival and migration $(7,31)$. It has been reported that overexpression of YAP may trigger transition of healthy epithelial cells into metastatic cells via epithelial-mesenchymal transition and may confer stem cell characteristics $(11,32,33)$.

A previous study demonstrated that YAP was associated with response to radiation-based treatments, and was predictive of poor recurrence-free survival in 86 patients with squamous cell carcinoma of the head and neck, including five LSCC cases (34). The present study revealed that compared with healthy surgical margins tissues, mRNA and protein expression levels of YAP were increased in 30 LSCC samples, as assessed by RT-qPCR and western blotting analysis, respectively. Positive expression of YAP was identified in 84/121 (69.4\%) of LSCC cases, and only 4/30 (13.3\%) of healthy tissues by IHC, and its subcellular localization was primarily accumulated in the nucleus with a reduced cytoplasmic presence in the majority of LSCC cases. 
To further identify clinicopathological characteristics and YAP expression levels in LSCC, 121 LSCC samples were examined by IHC. The results indicated that YAP protein expression levels were positively associated with clinical stage, TNM stage, LNM and differentiated degree, suggesting that YAP expression was directly associated with an aggressive phenotype. Therefore, YAP may serve a key role in the development and progression of LSCC. To identify a novel cancer biomarker in addition to standard TNM and histopathological grading to potentially assist post-surgery follow-up, YAP protein expression levels in 91 LSCC samples were examined with follow-up data. The expression of YAP was significantly associated with OS time. Patients with LSCC with positive YAP expression were more likely to have significantly poorer OS by univariate analysis and multivariate analysis. Therefore, elevated expression of YAP in LSCC may contribute to a poor prognostic phenotype. These findings suggested that YAP in conjunction with standard TNM and histopathological grading may be a more effective strategy to predict the prognosis of LSCC patients.

Similarly, overexpression of the YAP oncogene is a frequent occurrence in numerous common human cancers and is associated with poorer survival (35). Pei et al (17) demonstrated that high levels of nuclear YAP were associated with histological differentiation, TNM stage, metastasis and poor prognosis in human cholangiocarcinoma. Liu et al (15) revealed that positive expression of YAP was associated with tumor differentiation, higher primary tumor and/or regional lymph node stages in urothelial carcinoma, and that YAP was a strong and independent predictor of short overall survival in urothelial carcinoma patients. In non-small-cell lung cancer, Wang et al (16) reported that YAP expression was predictive of poor patient survival and was an independent prognostic indicator via multivariate analysis. Xia et al (18) additionally reported that YAP is significantly upregulated in human ovarian cancer tissues and is associated with patient prognosis.

In conclusion, the present study demonstrated that the expression level of YAP was significantly increased in LSCC and was associated with the malignant status of LSCC. Therefore, YAP may represent a potential novel prognostic factor for OS in LSCC. However, due to the limited patient sample size in the present study, further studies are required.

\section{Acknowledgements}

The present study was supported by the National Natural Science Foundation of China (grant nos. 81271078, 81300827 and 81500791). The authors would like to thank Professor Desheng Wang (Affiliated Union Hospital of Fujian Medical University, Fujian, China) and Professor Donghong Yu (The First Hospital Affiliated to Bengbu Medical College, Bengbu, China) for their useful comments.

\section{References}

1. Siegel R, Naishadham D and Jemal A: Cancer statistics, 2012. CA Cancer J Clin 62: 10-29, 2012.

2. Ferlay J, Parkin DM and Steliarova-Foucher E: Estimates of cancer incidence and mortality in Europe in 2008. Eur J Cancer 46: 765-781, 2010.

3. Cosetti M, Yu GP and Schantz SP: Five-year survival rates and time trends of laryngeal cancer in the US population. Arch Otolaryngol Head Neck Surg 134: 370-379, 2008.
4. Russo N, Wang X, Liu M, Banerjee R, Goto M, Scanlon C, Metwally T, Inglehart RC, Tsodikov A, Duffy S, et al: A novel approach to biomarker discovery in head and neck cancer using an autoantibody signature. Oncogene 32: 5026-5037, 2013.

5. Bai H, Gayyed MF, Lam-Himlin DM, Klein AP, Nayar SK, $\mathrm{Xu}$ Y, Khan M, Argani P, Pan D and Anders RA: Expression of yes-associated protein, YAP, modulates survivin expression in primary liver malignancies. Hum Pathol 43: 1376-1385, 2012.

6. Dong J, Feldmann G, Huang J, Wu S, Zhang N, Comerford SA, Gayyed MF, Anders RA, Maitra A and Pan D: Elucidation of a universal size-control mechanism in drosophila and mammals. Cell 130: 1120-1133, 2007.

7. Zhao B, Ye X, Yu J, Li L, Li W, Li S, Lin JD, Wang CY, Chinnaiyan AM, Lai ZC and Guan KL: TEAD mediates YAP-dependent gene induction and growth control. Genes Dev 22: 1962-1971, 2008.

8. Overholtzer M, Zhang J, Smolen GA, Muir B, Li W, Sgroi DC, Deng CX, Brugge JS and Haber DA: Transforming properties of YAP, a candidate oncogene on the chromosome 11q22 amplicon. Proc Natl Acad Sci USA 103: 12405-12410, 2006.

9. Steinhardt AA, Gayyed MF, Klein AP, Dong J, Maitra A, Pan D, Montgomery EA and Anders RA: Expression of Yes-associated protein in common solid tumors. Hum Pathol 39: 1582-1589, 2008.

10. Harvey KF, Zhang X and Thomas DM: The Hippo pathway and human cancer. Nat Rev Cancer 13: 246-257, 2013.

11. Moroishi T, Hansen CG and Guan KL: The emerging roles of YAP and TAZ in cancer. Nat Rev Cancer 15: 73-79, 2015.

12. Pan D: The hippo signaling pathway in development and cancer. Dev Cell 19: 491-505, 2010

13. Xu MZ, Yao TJ, Lee NP, Ng IO, Chan YT, Zender L, Lowe SW, Poon RT and Luk JM: Yes-associated protein is an independent prognostic marker in hepatocellular carcinoma. Cancer 115: 4576-4585, 2009.

14. Kang W, Tong JH, Chan AW, Lee TL, Lung RW, Leung PP, So KK, Wu K, Fan D, Yu J, et al: Yes-associated protein 1 exhibits oncogenic property in gastric cancer and its nuclear accumulation associates with poor prognosis. Clin Cancer Res 17: 2130-2139, 2011.

15. Liu JY, Li YH, Lin HX, Liao YJ, Mai SJ, Liu ZW, Zhang ZL, Jiang LJ, Zhang JX, Kung HF, et al: Overexpression of YAP 1 contributes to progressive features and poor prognosis of human urothelial carcinoma of the bladder. BMC Cancer 13: 349, 2013.

16. Wang Y, Dong Q, Zhang Q, Li Z, Wang E and Qiu X: Overexpression of yes-associated protein contributes to progression and poor prognosis of non-small-cell lung cancer. Cancer Sci 101: 1279-1285, 2010.

17. Pei T, Li Y, Wang J, Wang H, Liang Y, Shi H, Sun B, Yin D, Sun J, Song R, et al: YAP is a critical oncogene in human cholangiocarcinoma. Oncotarget 6: 17206-17220, 2015.

18. Xia Y, Chang T, Wang Y, Liu Y, Li W, Li M and Fan HY: YAP promotes ovarian cancer cell tumorigenesis and is indicative of a poor prognosis for ovarian cancer patients. PLoS One 9: e91770, 2014.

19. Muramatsu T, Imoto I, Matsui T, Kozaki K, Haruki S, Sudol M, Shimada Y, Tsuda H, Kawano $\mathrm{T}$ and Inazawa J: YAP is a candidate oncogene for esophageal squamous cell carcinoma. Carcinogenesis 32: 389-398, 2011.

20. Ge L, Smail M, Meng W, Shyr Y, Ye F, Fan KH, Li X, Zhou HM and Bhowmick NA: Yes-associated protein expression in head and neck squamous cell carcinoma nodal metastasis. PloS One 6: e27529, 2011

21. Wang DS, Pan CC, Lai HC and Huang JM: Expression of HMGA1 and ezrin in laryngeal squamous cell carcinoma. Acta Otolaryngol 133: 626-632, 2013.

22. Livak KJ and Schmittgen TD: Analysis of relative gene expression data using real-time quantitative PCR and the 2(-Delta Delta C(T)) Method. Methods 25: 402-408, 2001.

23. Wittekind C, Asamura $\mathrm{H}$ and Sobin LH (eds): Head and Neck Tumours. In: TNM Atlas: Illustrated Guide to the TNM Classification of Malignant Tumours. 6th Edition. John Wiley \& Sons, Ltd., Oxford, 2014.

24. Kleinsasser O: Revision of classification of laryngeal cancer, is it long overdue? (Proposals for an improved TN-classification). J Laryngol Otol 106: 197-204, 1992.

25. Mo JS, Park HW and Guan KL: The Hippo signaling pathway in stem cell biology and cancer. EMBO Rep 15: 642-656, 2014.

26. Varelas X, Miller BW, Sopko R, Song S, Gregorieff A, Fellouse FA, Sakuma R, Pawson T, Hunziker W, McNeill H, et al: The Hippo pathway regulates Wnt/beta-catenin signaling. Dev Cell 18: 579-591, 2010. 
27. Avruch J, Zhou D and Bardeesy N: YAP oncogene overexpression supercharges colon cancer proliferation. Cell Cycle 11: 1090-1096, 2012.

28. Hergovich A: YAP-Hippo signalling downstream of leukemia inhibitory factor receptor: Implications for breast cancer. Breast Cancer Res 14: 326, 2012.

29. He C, Mao D, Hua G, Lv X, Chen X, Angeletti PC, Dong J, Remmenga SW, Rodabaugh KJ, Zhou J, et al: The Hippo/YAP pathway interacts with EGFR signaling and HPV oncoproteins to regulate cervical cancer progression. EMBO Mol Med 7: 1426-1449, 2015

30. Lee KP, Lee JH, Kim TS, Kim TH, Park HD, Byun JS, Kim MC, Jeong WI, Calvisi DF, Kim JM and Lim DS: The Hippo-Salvador pathway restrains hepatic oval cell proliferation, liver size and liver tumorigenesis. Proc Natl Acad Sci USA 107: 8248-8253, 2010.

31. Hao Y, Chun A, Cheung K, Rashidi B and Yang X: Tumor suppressor LATS1 is a negative regulator of oncogene YAP. J Biol Chem 283: 5496-5509, 2008.
32. Chen D, Sun Y, Wei Y,Zhang P, Rezaeian AH, Teruya-Feldstein J, Gupta S, Liang H, Lin HK, Hung MC and Ma L: LIFR is a breast cancer metastasis suppressor upstream of the Hippo-YAP pathway and a prognostic marker. Nat Med 18: 1511-1517, 2012.

33. Shao DD, Xue W, Krall EB, Bhutkar A, Piccioni F, Wang X, Schinzel AC, Sood S, Rosenbluh J, Kim JW, et al: KRAS and YAP1 converge to regulate EMT and tumor survival. Cell 158: 171-184, 2014.

34. Akervall J, Nandalur S, Zhang J, Qian CN, Goldstein N, Gyllerup P, Gardinger Y, Alm J, Lorenc K, Nilsson K, et al: A novel panel of biomarkers predicts radioresistance in patients with squamous cell carcinoma of the head and neck. Eur J Cancer 50: 570-581, 2014.

35. Lu L, Li Y, Kim SM, Bossuyt W, Liu P, Qiu Q, Wang Y, Halder G, Finegold MJ, Lee JS and Johnson RL: Hippo signaling is a potent in vivo growth and tumor suppressor pathway in the mammalian liver. Proc Natl Acad Sci USA 107: 1437-1442, 2010. 